\title{
Coincidence theorems via alpha cuts of $L$-fuzzy sets with applications
}

\author{
Maliha Rashid ${ }^{1}$, Marwan Amin Kutbi ${ }^{2}$ and Akbar Azam³
}

\section{${ }^{*}$ Correspondence:}

akbarazam@yahoo.com

${ }^{3}$ Department of Mathematics, COMSATS Institute of Information

Technology, Chack Shahzad, Islamabad, 44000, Pakistan

Full list of author information is

available at the end of the article

\section{Springer}

\begin{abstract}
In the present paper, existence theorems of coincidence points of a crisp mapping and a sequence of L-fuzzy mappings have been established in a complete metric space under contractive type conditions in connection with newly defined notions of $D_{\alpha_{L}}$ and $d_{L}^{\infty}$ distances on the class of $L$-fuzzy sets. Furthermore, we obtain some fixed point theorems for L-fuzzy set-valued mappings to extend a variety of recent results on fixed points for fuzzy mappings and multivalued mappings in the literature. As applications, first we obtain coincidence points of a sequence of multivalued mappings with a self mapping and next established an existence and uniqueness theorem of the solution for a generalized class of nonlinear integral equations.
\end{abstract} MSC: $46 \mathrm{~S} 40 ; 47 \mathrm{H} 10 ; 54 \mathrm{H} 25$

Keywords: coincidence point; fixed point; multivalued mapping; L-fuzzy mapping; fuzzy mapping; integral equation

\section{Introduction}

Since his creation, man has always been making sincere efforts in understanding nature intelligently and then developing a powerful connection between life and its requirements. These efforts consist of three phases: understanding of the surrounding environment, acknowledgement of new things, and then planning for the future. In this search so many issues like linguistic interpretation, inaccurate judgment, characterization of interrelated phenomena into proper classifications, use of restricted techniques, vague analysis of results and many others, highly affect the accuracy of the results. The above mentioned hurdles related with interpretation of data can be tackled to a great extent by considering fuzzy sets (due to their flexible nature) in place of crisp sets.

After the discovery of fuzzy set by Zadeh [1] a great revolution arose in the field of analysis. The potential of the introduced notion was realized by research workers of different fields of science and technology. By introducing a contraction condition for fuzzy mappings Heilpern [2] generalized the Banach principle and established a fixed point theorem for fuzzy mappings in complete metric linear spaces. Afterwards, many authors, e.g., [3-16] generalized and extended this result in various directions. In [17] Edelstein extended the Banach contraction principle by using the idea of locally and globally contractive mappings. Subsequently, many authors, e.g., [18-22] utilized this concept to prove numerous results. In 1967, Goguen [23] initiated an interesting generalization of fuzzy sets namely called $L$-fuzzy sets. The concept of $L$-fuzzy set is superior to fuzzy sets as $L$ is a lattice which is not necessarily a totally ordered set. Recently, Rashid et al. [24] intro-

@2014 Rashid et al.; licensee Springer. This is an Open Access article distributed under the terms of the Creative Commons Attribution License (http://creativecommons.org/licenses/by/2.0), which permits unrestricted use, distribution, and reproduction in any medium, provided the original work is properly cited. 
duced the concept of $L$-fuzzy mappings and proved a common fixed point theorem via $\beta_{F_{L}}$-admissible pair of $L$-fuzzy mappings.

In this article we introduce the notions of $D_{\alpha_{L}}$ and $d_{L}^{\infty}$ distances for $L$-fuzzy sets to identify a contractive relation between $L$-fuzzy mappings and crisp mappings. Making use of this contractive relation on a complete metric space a coincidence point is obtained of a sequence of $L$-fuzzy mappings and a single valued crisp mapping. Analogous coincidence theorems for fuzzy mappings and multivalued mappings have been obtained as corollaries. These corollaries regarding coincidence point of fuzzy mappings and multivalued mappings have not been seen in the literature and therefore most of them are still original and new results. However, some imaginative fixed point theorems $[3,9,11,13,17,18,25$, $26]$ in the literature can be obtained as corollaries.

We also present some applications of the main theorem in two directions, one for obtaining fixed points and coincidence points of formal multivalued mappings and the other is for solutions of a generalized class of nonlinear integral equations to enhance the validity of our result.

\section{Preliminaries}

This section lists some preliminary notions and results. Let $(X, d)$ be a metric space, denote:

$C B(X)=\{A: A$ is nonempty closed and bounded subset of $X\}$,

$C(X)=\{A: A$ is nonempty compact subset of $X\}$.

For $\epsilon>0$ and the sets $A, B \in C B(X)$ define

$$
\begin{aligned}
& d(x, A)=\inf _{y \in A} d(x, y), \\
& d(A, B)=\inf _{x \in A, y \in B} d(x, y), \\
& N(\epsilon, A)=\{x \in X: d(x, a)<\epsilon, \text { for some } a \in A\}, \\
& E_{A, B}=\{\epsilon>0: A \subseteq N(\epsilon, B), B \subseteq N(\epsilon, A)\} .
\end{aligned}
$$

Then the Hausdorff metric $H$ on $C B(X)$ induced by $d$ is defined as

$$
H(A, B)=\inf E_{A, B} .
$$

A fuzzy set in $X$ is a function with domain $X$ and values in [0,1]. If $A$ is a fuzzy set and $x \in X$, then the function values $A(x)$ is called the grade of membership of $x$ in $A$. The $\alpha$-level set of $A$ is denoted by $[A]_{\alpha}$ and is defined as follows:

$$
[A]_{\alpha}=\{x: A(x) \geq \alpha\} \quad \text { if } \alpha \in(0,1]
$$

A fuzzy set $A$ in a metric linear space $V$ is said to be an approximate quantity if and only if $[A]_{\alpha}$ is compact and convex in $V$ for each $\alpha \in[0,1]$ and $\sup _{x \in V} A(x)=1$. The collection of all approximate quantities in $V$ is denoted by $W(V)$.

For $A, B \in I^{X}, A \subset B$ means $A(x) \leq B(x)$ for each $x \in X$. If there exists an $\alpha \in[0,1]$ such that $[A]_{\alpha},[B]_{\alpha} \in C B(X)$ then define

$$
D_{\alpha}(A, B)=H\left([A]_{\alpha},[B]_{\alpha}\right) .
$$


If $[A]_{\alpha},[B]_{\alpha} \in C B(X)$ for each $\alpha \in[0,1]$ then define

$$
d_{\infty}(A, B)=\sup _{\alpha} D_{\alpha}(A, B)
$$

Lemma 2.1 [26] Let $(X, d)$ be a metric space and $A, B \in C B(X)$, then for each $a \in A$

$$
d(a, B) \leq H(A, B)
$$

Lemma 2.2 [26] Let $(X, d)$ be a metric space and $A, B \in C B(X)$, then for each $a \in A, \epsilon>0$, there exists an element $b \in B$ such that

$$
d(a, b) \leq H(A, B)+\epsilon .
$$

Definition 2.3 [23] A partially ordered set $(L, \precsim L)$ is called

(i) a lattice, if $a \vee b \in L, a \wedge b \in L$ for any $a, b \in L$;

(ii) a complete lattice, if $\vee A \in L, \wedge A \in L$ for any $A \subseteq L$;

(iii) distributive if $a \vee(b \wedge c)=(a \vee b) \wedge(a \vee c), a \wedge(b \vee c)=(a \wedge b) \vee(a \wedge c)$ for any $a, b, c \in L$.

Definition 2.4 [23] Let $L$ be a lattice with top element $1_{L}$ and bottom element $0_{L}$ and let $a, b \in L$. Then $b$ is called a complement of $a$, if $a \vee b=1_{L}$, and $a \wedge b=0_{L}$. If $a \in L$ has a complement element, then it is unique. It is denoted by $a$.

Definition 2.5 [23] A $L$-fuzzy set $A$ on a nonempty set $X$ is a function $A: X \rightarrow L$, where $L$ is complete distributive lattice with $1_{L}$ and $0_{L}$.

Remark 2.6 The class of $L$-fuzzy sets is larger than the class of fuzzy sets as an $L$-fuzzy set is a fuzzy set if $L=[0,1]$.

From now, the class of $L$-fuzzy subsets of $X$ will be denoted by $F_{L}(X)$. The $\alpha_{L}$-level set of $L$-fuzzy set $A$, is denoted by $A_{\alpha_{L}}$, and is defined as follows:

$$
\begin{aligned}
& A_{\alpha_{L}}=\left\{x: \alpha_{L} \precsim{ }_{L} A(x)\right\} \quad \text { if } \alpha_{L} \in L \backslash\left\{O_{L}\right\}, \\
& A_{O_{L}}=\overline{\left\{x: O_{L} \precsim L A(x)\right\} .}
\end{aligned}
$$

Here $\bar{B}$ denotes the closure of the set $B$.

Definition 2.7 [24] Let $X$ be an arbitrary set, $Y$ be a metric space. A mapping $T$ is called $L$-fuzzy mapping if $T$ is a mapping from $X$ into $F_{L}(Y)$ (class of $L$-fuzzy subsets of $Y$ ). A $L$-fuzzy mapping $T$ is a $L$-fuzzy subset on $X \times Y$ with membership function $T(x)(y)$. The function $T(x)(y)$ is the grade of membership of $y$ in $T(x)$.

Definition 2.8 [24] Let $(X, d)$ be a metric space and $S, T$ be $L$-fuzzy mappings from $X$ into $F_{L}(X)$. A point $z \in X$ is called a $L$-fuzzy fixed point of $T$ if $z \in[T z]_{\alpha_{L}}$, for some $\alpha_{L} \in L \backslash\left\{O_{L}\right\}$. The point $z \in X$ is called a common $L$-fuzzy fixed point of $S$ and $T$ if $z \in[S z]_{\alpha_{L}} \cap[T z]_{\alpha_{L}}$. When $\alpha_{L}=1_{L}$, it is called a common fixed point of $L$-fuzzy mappings. 
Definition 2.9 Let $\varepsilon \in(0, \infty]$, and $\lambda \in(0,1)$. A metric space $(X, d)$ is said to be $\varepsilon$-chainable if given $u, v \in X$, there exists an $\varepsilon$-chain from $u$ to $v$ (i.e., a finite set of points $u=u_{0}$, $u_{1}, u_{2}, \ldots, u_{l}=v$ such that $d\left(u_{t-1}, u_{t}\right)<\varepsilon$ for all $\left.t=1,2, \ldots, l\right)$.

Definition 2.10 [8] A function $\varphi:[0,+\infty) \rightarrow[0,1)$ is said to be a $M T$-function if it satisfies the following condition:

$\lim _{r \rightarrow t^{+}} \phi(r)<1 \quad$ for all $t \in[0,+\infty)$.

Clearly, if $\varphi:[0,+\infty) \rightarrow[0,1)$ is a nondecreasing function or a nonincreasing function, then it is a $M T$-function. So the set of $M T$-functions is a rich class.

Proposition 2.11 [8] Let $\varphi:[0,+\infty) \rightarrow[0,1)$ be a function. Then the following statements are equivalent.

(i) $\varphi$ is a MT-function.

(ii) For each $t \in[0, \infty)$, there exist $r_{t}^{(1)} \in[0,1)$ and $\varepsilon_{t}^{(1)}>0$ such that $\varphi(s) \leq r_{t}^{(1)}$ for all $s \in\left(t, t+\varepsilon_{t}^{(1)}\right)$.

(iii) For each $t \in[0, \infty)$, there exist $r_{t}^{(2)} \in[0,1)$ and $\varepsilon_{t}^{(2)}>0$ such that $\varphi(s) \leq r_{t}^{(2)}$ for all $s \in\left[t, t+\varepsilon_{t}^{(2)}\right]$.

(iv) For each $t \in[0, \infty)$, there exist $r_{t}^{(3)} \in[0,1)$ and $\varepsilon_{t}^{(3)}>0$ such that $\varphi(s) \leq r_{t}^{(3)}$ for all $s \in\left(t, t+\varepsilon_{t}^{(3)}\right]$.

(v) For each $t \in[0, \infty)$, there exist $r_{t}^{(4)} \in[0,1)$ and $\varepsilon_{t}^{(4)}>0$ such that $\varphi(s) \leq r_{t}^{(4)}$ for all $s \in\left[t, t+\varepsilon_{t}^{(4)}\right)$.

(vi) For any nonincreasing sequence $\left\{x_{n}\right\}_{n \in \mathbb{N}}$ in $[0, \infty)$, we have $0 \leq \sup _{n \in \mathbb{N}} \varphi\left(x_{n}\right)<1$.

(vii) $\varphi$ is a function of contractive factor [27], that is, for any strictly decreasing sequence $\left\{x_{n}\right\}_{n \in \mathbb{N}}$ in $[0, \infty)$, we have $0 \leq \sup _{n \in \mathbb{N}} \varphi\left(x_{n}\right)<1$.

\section{Coincidence theorems for L-fuzzy mappings}

In this section the notion of $D_{\alpha_{L}}$ distance is used to study coincidence theorems concerning $L$-fuzzy mappings. For a metric space $(X, d)$, we define

$$
D_{\alpha_{L}}(A, B)=H\left(A_{\alpha_{L}}, B_{\alpha_{L}}\right)
$$

and

$$
d_{L}^{\infty}(A, B)=\sup _{\alpha_{L} \in L \backslash\left\{O_{L}\right\}} D_{\alpha_{L}}(A, B)
$$

whenever $A, B \in F_{L}(X)$ and $A_{\alpha_{L}}, B_{\alpha_{L}} \in C B(X)$ for each $\alpha_{L} \in L \backslash\left\{O_{L}\right\}$.

Definition 3.1 A mapping $T: X \rightarrow X$ is called an $(\varepsilon, \lambda)$ uniformly locally contractive mapping if $u, v \in X$ and $0<d(u, v)<\varepsilon$ implies $d(T u, T v) \leq \lambda d(u, v)$. A mapping $T: X \rightarrow F_{L}(X)$ is called an $(\varepsilon, \lambda)$ uniformly locally contractive $L$-fuzzy mapping if $u, v \in X$ and $0<d(u, v)<\varepsilon$, then $d_{L}^{\infty}(T(u), T(v)) \leq \lambda d(u, v)$.

Theorem 3.2 Let $\varepsilon \in(0, \infty],(X, d)$ be a complete $\varepsilon$-chainable metric space, $\left\{T_{q}\right\}_{q=1}^{\infty}$ a sequence of mappings from $X$ into $F_{L}(X)$, and $S: X \rightarrow X$ a surjection such that for each $u \in X$ and $q \in \mathbb{N},\left[T_{q}(u)\right]_{\alpha_{L}} \in C B(X)$, for some $\alpha_{L} \in L \backslash\left\{0_{L}\right\}$. If $u, v \in X$ such that $0<d(S u, S v)<\varepsilon$ 
implies

$$
D_{\alpha_{L}}\left(T_{q}(u), T_{r}(v)\right) \leq \mu(d(S u, S v)) d(S u, S v)
$$

for all $q, r \in \mathbb{N}$, where $\mu:[0, \varepsilon) \rightarrow[0,1)$ is a MT-function, then $S$ and the sequence $\left\{T_{q}\right\}_{q=1}^{\infty}$ have a coincidence point, i.e., there exists $v^{*} \in X$ such that $S v^{*} \in \bigcap_{q \in \mathbb{N}}\left[T_{q}\left(v^{*}\right)\right]_{\alpha_{L}}$.

Proof Let $v_{0}$ be an arbitrary, but fixed element of $X$. Find $v_{1} \in X$ such that $S v_{1} \in\left[T_{1}\left(v_{0}\right)\right]_{\alpha_{L}}$. Let

$$
S v_{0}=S u_{(1,0)}, S u_{(1,1)}, S u_{(1,2)}, \ldots, S u_{(1, l)}=S v_{1} \in\left[T_{1}\left(v_{0}\right)\right]_{\alpha_{L}}
$$

be an arbitrary $\varepsilon$-chain from $S v_{0}$ to $S v_{1}$. (Without any loss of generality, we assume that $S u_{(1, q)} \neq S u_{(1, r)}$ for each $q, r \in\{0,1,2, \ldots, l\}$ with $q \neq r$.)

Since $0<d\left(S u_{(1,0)}, S u_{(1,1)}\right)<\varepsilon$, we get

$$
\begin{aligned}
D_{\alpha_{L}}\left(T_{1}\left(u_{(1,0)}\right), T_{2}\left(u_{(1,1)}\right)\right) & \leq \mu\left(d\left(S u_{(1,0)}, S u_{(1,1)}\right)\right) d\left(S u_{(1,0)}, S u_{(1,1)}\right) \\
& <\sqrt{\mu\left(d\left(S u_{(1,0)}, S u_{(1,1)}\right)\right)} d\left(S u_{(1,0)}, S u_{(1,1)}\right) \\
& <d\left(S u_{(1,0)}, S u_{(1,1)}\right)<\varepsilon .
\end{aligned}
$$

Rename $S v_{1}$ as $S u_{(2,0)}$. Since $S u_{(2,0)} \in\left[T_{1}\left(u_{(1,0)}\right)\right]_{\alpha_{L}}$, using Lemma 2.1 we find $S u_{(2,1)} \in$ $\left[T_{2}\left(u_{(1,1)}\right)\right]_{\alpha_{L}}$ such that

$$
\begin{aligned}
d\left(S u_{(2,0)}, S u_{(2,1)}\right) & <\sqrt{\mu\left(d\left(S u_{(1,0)}, S u_{(1,1)}\right)\right)} d\left(S u_{(1,0)}, S u_{(1,1)}\right) \\
& <d\left(S u_{(1,0)}, S u_{(1,1)}\right)<\varepsilon .
\end{aligned}
$$

Since $0<d\left(S u_{(1,1)}, S u_{(1,2)}\right)<\varepsilon$, we deduce that

$$
\begin{aligned}
D_{\alpha_{L}}\left(T_{2}\left(u_{(1,1)}\right), T_{2}\left(u_{(1,2)}\right)\right) & \leq \mu\left(d\left(\operatorname{Su}_{(1,1)}, S u_{(1,2)}\right)\right) d\left(S u_{(1,1)}, S u_{(1,2)}\right) \\
& <\sqrt{\mu\left(d\left(S u_{(1,1)}, S u_{(1,2)}\right)\right)} d\left(S u_{(1,1)}, S u_{(1,2)}\right) \\
& <d\left(S u_{(1,1)}, S u_{(1,2)}\right)<\varepsilon .
\end{aligned}
$$

Similarly to $S u_{(2,1)} \in\left[T_{2}\left(u_{(1,1)}\right)\right]_{\alpha_{L}}$, again using Lemma 2.1 we find $S u_{(2,2)} \in\left[T_{2}\left(u_{(1,2)}\right)\right]_{\alpha_{L}}$ such that

$$
\begin{aligned}
d\left(S u_{(2,1)}, S u_{(2,2)}\right) & <\sqrt{\mu\left(d\left(S u_{(1,1)}, S u_{(1,2)}\right)\right)} d\left(S u_{(1,1)}, S u_{(1,2)}\right) \\
& <d\left(S u_{(1,1)}, S u_{(1,2)}\right)<\varepsilon .
\end{aligned}
$$

Thus we obtain a set $\left\{S u_{(2,0)}, S u_{(2,1)}, S u_{(2,2)}, \ldots, S u_{(2, l)}\right\}$ of $l+1$ points of $X$ such that $S u_{(2,0)} \in$ $\left[T_{1}\left(u_{(1,0)}\right)\right]_{\alpha_{L}}$ and $S u_{(2, t)} \in\left[T_{2}\left(u_{(1, t)}\right)\right]_{\alpha_{L}}$ for $t=1,2, \ldots, l$, with

$$
\begin{aligned}
d\left(\operatorname{Su}_{(2, t)}, S u_{(2, t+1)}\right) & <\sqrt{\mu\left(d\left(\operatorname{Su}_{(1, t)}, S u_{(1, t+1)}\right)\right)} d\left(S u_{(1, t)}, S u_{(1, t+1)}\right) \\
& <d\left(S u_{(1, t)}, S u_{(1, t+1)}\right)<\varepsilon
\end{aligned}
$$

for $t=0,1,2, \ldots, l-1$. 
Let $S u_{(2, l)}=v_{2}$. Thus the set of points $S v_{1}=S u_{(2,0)}, S u_{(2,1)}, S u_{(2,2)}, \ldots, S u_{(2, l)}=S v_{2} \in$ $\left[T_{2}\left(v_{1}\right)\right]_{\alpha_{L}}$ is an $\varepsilon$-chain from $S v_{1}$ to $S v_{2}$. Rename $S v_{2}$ as $S u_{(3,0)}$. Then by the same procedure we obtain an $\varepsilon$-chain

$$
S v_{2}=S u_{(3,0)}, S u_{(3,1)}, S u_{(3,2)}, \ldots, S u_{(3, l)}=S v_{3} \in\left[T_{3}\left(v_{2}\right)\right]_{\alpha_{L}}
$$

from $S v_{2}$ to $S v_{3}$. Inductively, we obtain

$$
S v_{h}=S u_{(h+1,0)}, S u_{(h+1,1)}, S u_{(h+1,2)}, \ldots, S u_{(h+1, l)}=S v_{h+1} \in\left[T_{h+1}\left(v_{h}\right)\right]_{\alpha_{L}}
$$

with

$$
\begin{aligned}
d\left(S u_{(h+1, t)}, S u_{(h+1, t+1)}\right) & <\sqrt{\mu\left(d\left(S u_{(h, t)}, S u_{(h, t+1)}\right)\right)} d\left(S u_{(h, t)}, S u_{(h, t+1)}\right) \\
& <d\left(S u_{(h, t)}, S u_{(h, t+1)}\right)<\varepsilon
\end{aligned}
$$

for $t=0,1,2, \ldots, l-1$.

Consequently, we construct a sequence $\left\{S v_{h}\right\}_{h=1}^{\infty}$ of points of $X$ with

$$
\begin{aligned}
& S v_{1}=S u_{(1, l)}=S u_{(2,0)} \in\left[T_{1}\left(v_{0}\right)\right]_{\alpha_{L}}, \\
& S v_{2}=S u_{(2, l)}=S u_{(3,0)} \in\left[T_{2}\left(v_{1}\right)\right]_{\alpha_{L}}, \\
& S v_{3}=S u_{(3, l)}=S u_{(4,0)} \in\left[T_{3}\left(v_{2}\right)\right]_{\alpha_{L}}, \\
& \vdots \\
& S v_{h+1}=S u_{(h+1, l)}=S u_{(h+2,0)} \in\left[T_{h+1}\left(v_{h}\right)\right]_{\alpha_{L}},
\end{aligned}
$$

for all $h \in \mathbb{N}$.

For each $t \in\{0,1,2, \ldots, l-1\}$, we deduce from (2) that $\left\{d\left(S u_{(h, t)}, S u_{(h, t+1)}\right)\right\}_{h=1}^{\infty}$ is a decreasing sequence of non-negative real numbers and therefore there exists $l_{t} \geq 0$ such that

$$
\lim _{h \rightarrow \infty} d\left(S u_{(h, t)}, S u_{(h, t+1)}\right)=l_{t}
$$

By assumption, $\limsup _{t \rightarrow l_{t}^{+}} \mu(t)<1$, so there exists $h_{t} \in \mathbb{N}$ such that $\mu\left(d\left(S u_{(h, t)}, S u_{(h, t+1)}\right)\right)<$ $\omega\left(l_{t}\right)$ (a non-negative real number) for all $h \geq h_{t}$ where $\lim \sup _{t \rightarrow l_{t}^{+}} \mu(t)<\omega\left(l_{t}\right)<1$.

Let

$$
\Theta_{t}=\max \left\{\max _{q=1, \ldots, h_{t}} \sqrt{\mu\left(d\left(S u_{(q, t)}, S u_{(q, t+1)}\right)\right)}, \sqrt{\omega\left(l_{t}\right)}\right\}
$$

Then, for every $h>h_{t}$, we obtain

$$
\begin{aligned}
d\left(S u_{(h, t)}, S u_{(h, t+1)}\right) & <\sqrt{\mu\left(d\left(S u_{(h-1, t)}, S u_{(h-1, t+1)}\right)\right)} d\left(S u_{(h-1, t)}, S u_{(h-1, t+1)}\right) \\
& <\sqrt{\omega\left(l_{t}\right)} d\left(S u_{(h-1, t)}, S u_{(h-1, t+1)}\right) \\
& \leq \Theta_{t} d\left(S u_{(h-1, t)}, S u_{(h-1, t+1)}\right) \\
& \leq\left(\Theta_{t}\right)^{2} d\left(S u_{(h-2, t)}, S u_{(h-2, t+1)}\right)
\end{aligned}
$$




$$
\begin{aligned}
& \leq \cdots \\
& \leq\left(\Theta_{t}\right)^{h-1} d\left(S u_{(1, t)}, S u_{(1, t+1)}\right) .
\end{aligned}
$$

Putting $N=\max \left\{h_{t}: t=0,1,2, \ldots, l-1\right\}$, we have

$$
\begin{aligned}
d\left(S v_{h-1}, S v_{h}\right) & =d\left(S u_{(h, 0)}, S u_{(h, l)}\right) \leq \sum_{t=0}^{l-1} d\left(S u_{(h, t)}, S u_{(h, t+1)}\right) \\
& <\sum_{t=0}^{l-1}\left(\Theta_{t}\right)^{h-1} d\left(S u_{(1, t)}, S u_{(1, t+1)}\right),
\end{aligned}
$$

for all $h>N+1$. Hence

$$
\begin{aligned}
d\left(S v_{h}, S v_{p}\right) & \leq d\left(S v_{h}, S v_{h+1}\right)+d\left(S v_{h+1}, S v_{h+2}\right)+\cdots+d\left(S v_{p-1}, S v_{p}\right) \\
& <\sum_{t=0}^{l-1}\left(\Theta_{t}\right)^{h} d\left(S u_{(1, t)}, S u_{(1, t+1)}\right)+\cdots+\sum_{t=0}^{l-1}\left(\Theta_{t}\right)^{p-1} d\left(S u_{(1, t)}, S u_{(1, t+1)}\right),
\end{aligned}
$$

whenever $p>h>N+1$.

Since $\Theta_{t}<1$ for all $t \in\{0,1,2, \ldots, l-1\}$, it follows that $\left\{S v_{h}=S u_{(h, l)}\right\}$ is a Cauchy sequence. Since $(X, d)$ is complete, there is $v^{*} \in X$ such that $S v_{h} \rightarrow S v^{*}$. Hence there exists an integer $M>0$ such that $h>M$ implies $d\left(S v_{h}, S v^{*}\right)<\varepsilon$. This from the point of view of inequality (1) implies $D_{\alpha_{L}}\left(T_{h+1}\left(v_{h}\right), T_{q}\left(v^{*}\right)\right)<\varepsilon$ for all $q \in \mathbb{N}$.

Now consider for all $q \in \mathbb{N}$,

$$
\begin{aligned}
d\left(S v^{*},\left[T_{q}\left(v^{*}\right)\right]_{\alpha_{L}}\right) & \leq d\left(S v^{*}, S v_{h+1}\right)+d\left(S v_{h+1},\left[T_{q}\left(v^{*}\right)\right]_{\alpha_{L}}\right) \\
& \leq d\left(S v^{*}, S v_{h+1}\right)+H\left(\left[T_{h+1}\left(v_{h}\right)\right]_{\alpha_{L}},\left[T_{q}\left(v^{*}\right)\right]_{\alpha_{L}}\right) \\
& \leq d\left(S v^{*}, S v_{h+1}\right)+D_{\alpha_{L}}\left(T_{h+1}\left(v_{h}\right), T_{q}\left(v^{*}\right)\right) \\
& \leq d\left(S v^{*}, S v_{h+1}\right)+\mu\left(d\left(S v_{h}, S v^{*}\right)\right) d\left(S v_{h}, S v^{*}\right) .
\end{aligned}
$$

Letting $h \rightarrow \infty$ in the above inequality, we get $d\left(S v^{*},\left[T_{q}\left(v^{*}\right)\right]_{\alpha_{L}}\right) \rightarrow 0$, which implies $S v^{*} \in$ $\left[T_{q}\left(v^{*}\right)\right]_{\alpha_{L}}$ for all $q \in \mathbb{N}$. Hence, $S v^{*} \in \bigcap_{q \in \mathbb{N}}\left[T_{q}\left(v^{*}\right)\right]_{\alpha_{L}}$.

Corollary 3.3 Let $\varepsilon \in(0, \infty],(X, d)$ a complete $\varepsilon$-chainable metric space, $\left\{T_{q}\right\}_{q=1}^{\infty}$ a sequence of mappings from $X$ into $F_{L}(X)$ and $S: X \rightarrow X$ a surjection such that for each $u \in X$ and $q \in \mathbb{N},\left[T_{q}(u)\right]_{\alpha_{L}} \in C B(X)$, for some $\alpha_{L} \in L \backslash\left\{0_{L}\right\}$. If $u, v \in X$ such that $0<d(S u, S v)<\varepsilon$, implies

$$
D_{\alpha_{L}}\left(T_{q}(u), T_{r}(v)\right) \leq \rho d(S u, S v)
$$

for all $q, r \in \mathbb{N}$, where $\rho \in(0,1)$, then $S$ and sequence $\left\{T_{q}\right\}_{q=1}^{\infty}$ have a coincidence point, i.e., there exists $v^{*} \in X$ such that $S v^{*} \in \bigcap_{q \in \mathbb{N}}\left[T_{q}\left(v^{*}\right)\right]_{\alpha_{L}}$.

Proof Apply Theorem 3.2 where $\mu$ is the $M T$-function defined as $\mu(t)=\rho$ for all $t \in$ $[0, \varepsilon)$.

In the following we furnish an example to support Theorem 3.2. 
Example 3.4 Let $\varepsilon \in(0, \infty], X=[0,1]$, and $d(u, v)=|u-v|$, whenever $u, v \in X$, then $(X, d)$ is a complete $\varepsilon$-chainable metric space. Let $L=\{\zeta, \eta, \xi, \varsigma\}$ with $\zeta \precsim_{L} \eta \precsim_{L} \zeta, \zeta \precsim_{L} \xi \precsim_{L} \varsigma$, $\eta$ and $\xi$ are not comparable, then $(L, \precsim L)$ is a complete distributive lattice. Suppose $\left\{T_{q}\right\}_{q=1}^{\infty}$ to be a sequence of mappings defined from $X$ into $F_{L}(X)$ as

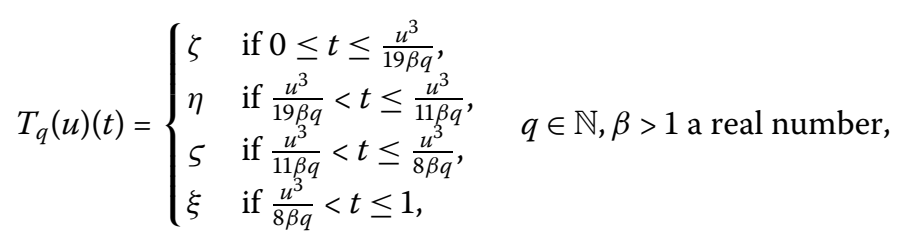

and $S: X \rightarrow X$ be a surjective self mapping defined as $S x=x^{3}$, for all $x \in X$. Now for $x, y \in$ $X \backslash\{0\}$, suppose $\varepsilon=\frac{1}{|x-y|} \in(0, \infty]$,

$$
0<d(S x, S y)=\left|x^{3}-y^{3}\right|=|x-y|\left|x^{2}+x y+y^{2}\right|<|x-y|<\varepsilon .
$$

Assume $\alpha_{L}=\eta$ then $\left[T_{q}(u)\right]_{\eta}=\left[0, \frac{u^{3}}{11 \beta q}\right]$. For $q, r \in \mathbb{N}$ with $q \leq r, u, v \in X$, and $\mu(t)=\frac{1}{\beta}$ for all $t \in(0, \varepsilon]$ consider

$$
\begin{aligned}
D_{\alpha_{L}}\left(T_{q}(u), T_{r}(v)\right) & =H\left(\left[T_{q}(u)\right]_{\alpha_{L}},\left[T_{r}(v)\right]_{\alpha_{L}}\right) \\
& =H\left(\left[0, \frac{u^{3}}{11 \beta q}\right],\left[0, \frac{v^{3}}{11 \beta r}\right]\right) \\
& =\frac{1}{11 \beta}\left|\frac{u^{3}}{q}-\frac{v^{3}}{r}\right| \\
& \leq \frac{1}{11 \beta q}\left|u^{3}-v^{3}\right| \\
& \leq \frac{1}{\beta}\left|u^{3}-v^{3}\right| \\
& =\mu(d(S u, S v)) d(S u, S v) .
\end{aligned}
$$

Since all the conditions of Theorems 3.2 are satisfied, there exist a coincidence point of $S$ and the sequence $\left\{T_{q}\right\}_{q=1}^{\infty}$, i.e.

$$
S 0=0 \in \bigcap_{q \in \mathbb{N}}\left[T_{q}(0)\right]_{\alpha_{L}}
$$

for some $\alpha_{L} \in L \backslash\left\{0_{L}\right\}$.

\section{Coincidence theorems for $L$-fuzzy mappings via $d_{L}^{\infty}$-distance}

This section deals with the study of coincidence theorems in connection with the notion of $d_{L}^{\infty}$-distance. The results proved in this section are also new.

Theorem 4.1 Let $\varepsilon \in(0, \infty],(X, d)$ a complete $\varepsilon$-chainable metric space, $\left\{T_{q}\right\}_{q=1}^{\infty}$ a sequence of L-fuzzy mappings from $X$ into $F_{L}(X)$ and $S: X \rightarrow X$ a surjection such that for each $u \in X$ and $q \in \mathbb{N},\left[T_{q}(u)\right]_{\alpha_{L}} \in C B(X)$, for some $\alpha_{L} \in L \backslash\left\{0_{L}\right\}$. If $u, v \in X$ such that $0<d(S u, S v)<\varepsilon$ implies

$$
d_{L}^{\infty}\left(T_{q}(u), T_{r}(v)\right) \leq \mu(d(S u, S v)) d(S u, S v)
$$


for all $q, r \in \mathbb{N}$, where $\mu:[0, \varepsilon) \rightarrow[0,1)$ is a MT-function, then $S$ and sequence $\left\{T_{q}\right\}_{q=1}^{\infty}$ have a coincidence point, i.e., there exists $v^{*} \in X$ such that $S v^{*} \in \bigcap_{q \in \mathbb{N}}\left[T_{q}\left(v^{*}\right)\right]_{\alpha_{L}}$.

Proof Since $D_{\alpha_{L}}\left(T_{q}(u), T_{r}(v)\right) \leq d_{L}^{\infty}\left(T_{q}(u), T_{r}(v)\right)$ for all $q, r \in \mathbb{N}$, the result follows immediately from Theorem 3.2.

By taking $S=I$ and in Theorem 4.1 we obtain the following result.

Corollary 4.2 Let $\varepsilon \in(0, \infty],(X, d)$ a complete $\varepsilon$-chainable metric space and $\left\{T_{q}\right\}_{q=1}^{\infty} a$ sequence of L-fuzzy mappings from $X$ into $F_{L}(X)$ such that for each $u \in X$ and $q \in \mathbb{N}$, $\left[T_{q}(u)\right]_{\alpha_{L}} \in C B(X)$, for some $\alpha_{L} \in L \backslash\left\{0_{L}\right\}$. If $u, v \in X$ such that $0<d(u, v)<\varepsilon$, implies

$$
d_{L}^{\infty}\left(T_{q}(u), T_{r}(v)\right) \leq \mu(d(u, v)) d(u, v)
$$

for all $q, r \in \mathbb{N}$, where $\mu:[0, \varepsilon) \rightarrow[0,1)$ is a MT-function, then the sequence $\left\{T_{q}\right\}_{q=1}^{\infty}$ has a common fixed point, i.e., there exists $v^{*} \in X$ such that $v^{*} \in \bigcap_{q \in \mathbb{N}}\left[T_{q}\left(v^{*}\right)\right]_{\alpha}$.

\section{Coincidence theorems for fuzzy mappings}

In the present section, by considering $L=[0,1]$ in Theorem 3.2, some further new results for fuzzy mappings are obtained.

Theorem 5.1 Let $\varepsilon \in(0, \infty],(X, d)$ a complete $\varepsilon$-chainable metric space, $\left\{T_{q}\right\}_{q=1}^{\infty}$ a sequence of fuzzy mappings from $X$ into $I^{X}$ and $S: X \rightarrow X$ a surjection such that for each $u \in X$ and $q \in \mathbb{N},\left[T_{q}(u)\right]_{\alpha} \in C B(X)$, for some $\alpha \in(0,1]$. If $u, v \in X$ such that $0<d(S u, S v)<\varepsilon$, implies

$$
D_{\alpha}\left(T_{q}(u), T_{r}(v)\right) \leq \mu(d(S u, S v)) d(S u, S v)
$$

for all $q, r \in \mathbb{N}$, where $\mu:[0, \varepsilon) \rightarrow[0,1)$ is a MT-function, then $S$ and sequence $\left\{T_{q}\right\}_{q=1}^{\infty}$ have a coincidence point, i.e., there exists $v^{*} \in X$ such that $S v^{*} \in \bigcap_{q \in \mathbb{N}}\left[T_{q}\left(v^{*}\right)\right]_{\alpha}$.

Corollary 5.2 Let $\varepsilon \in(0, \infty],(X, d)$ a complete $\varepsilon$-chainable metric linear space, $\left\{T_{q}\right\}_{q=1}^{\infty}$ a sequence offuzzy mappings from $X$ into $W(X)$ and $S: X \rightarrow X$ a surjection such that for each $u \in X$ and $q \in \mathbb{N},\left[T_{q}(u)\right]_{\alpha} \in C B(X)$, for some $\alpha \in(0,1]$. If $u, v \in X$ such that $0<d(S u, S v)<\varepsilon$, implies

$$
d_{\infty}\left(T_{q}(u), T_{r}(v)\right) \leq \mu(d(S u, S v)) d(S u, S v)
$$

for all $q, r \in \mathbb{N}$, where $\mu:[0, \varepsilon) \rightarrow[0,1)$ is a MT-function, then $S$ and sequence $\left\{T_{q}\right\}_{q=1}^{\infty}$ have a coincidence point, i.e., there exists $v^{*} \in X$ such that $S v^{*} \in \bigcap_{q \in \mathbb{N}}\left[T_{q}\left(v^{*}\right)\right]_{\alpha}$.

Proof Since $W(X) \subseteq C B(X)$ and $D_{\alpha}\left(T_{q}(u), T_{r}(v)\right) \leq d_{\infty}\left(T_{q}(u), T_{r}(v)\right)$ for all $q, r \in \mathbb{N}$, the result follows immediately from Theorem 5.1.

\section{Fixed point theorems for L-fuzzy mappings}

In this section some new fixed point results are deduced from the above mentioned coincidence results. If we take $S=I$ in Theorem 3.2 we get the following result. 
Theorem 6.1 Let $\varepsilon \in(0, \infty],(X, d)$ a complete $\varepsilon$-chainable metric space and $\left\{T_{q}\right\}_{q=1}^{\infty}$ a sequence of mappings from $X$ into $F_{L}(X)$ such that for each $u \in X$ and $q \in \mathbb{N},\left[T_{q}(u)\right]_{\alpha_{L}} \in$ $C B(X)$, for some $\alpha_{L} \in L \backslash\left\{0_{L}\right\}$. If $u, v \in X$ such that $0<d(u, v)<\varepsilon$ implies

$$
D_{\alpha_{L}}\left(T_{q}(u), T_{r}(v)\right) \leq \mu(d(u, v)) d(u, v)
$$

for all $q, r \in \mathbb{N}$, where $\mu:[0, \varepsilon) \rightarrow[0,1)$ is a MT-function, then the sequence $\left\{T_{q}\right\}_{q=1}^{\infty}$ has a common fixed point, i.e., there exists $v^{*} \in X$ such that $v^{*} \in \bigcap_{q \in \mathbb{N}}\left[T_{q}\left(v^{*}\right)\right]_{\alpha_{L}}$.

If we take $S=I$ in Theorem 5.1 we get the following result.

Corollary 6.2 Let $\varepsilon \in(0, \infty],(X, d)$ a complete $\varepsilon$-chainable metric space and $\left\{T_{q}\right\}_{q=1}^{\infty} a$ sequence of mappings from $X$ into $I^{X}$ such that for each $u \in X$ and $q \in \mathbb{N},\left[T_{q}(u)\right]_{\alpha} \in C B(X)$, for some $\alpha \in(0,1]$. If $u, v \in X$ such that $0<d(u, v)<\varepsilon$, implies

$$
D_{\alpha}\left(T_{q}(u), T_{r}(v)\right) \leq \mu(d(u, v)) d(u, v)
$$

for all $q, r \in \mathbb{N}$, where $\mu:[0, \varepsilon) \rightarrow[0,1)$ is a MT-function, then the sequence $\left\{T_{q}\right\}_{q=1}^{\infty}$ has a common fixed point, i.e., there exists $v^{*} \in X$ such that $v^{*} \in \bigcap_{q \in \mathbb{N}}\left[T_{q}\left(v^{*}\right)\right]_{\alpha}$.

By considering $\alpha=1$ in the above corollary we deduce the main result and hence all the corollaries of [18].

\section{Applications to multivalued maps}

Multivalued mapping is a left-total relation, arise in optimal control theory and game theory. In mathematics, multivalued mappings play an increasingly important role. For example fixed point results for multivalued mappings have been applied to prove existence of Nash equilibrium, the solutions of integral and differential inclusions etc. In this section, we will apply our main result to prove some coincidence results for multivalued mappings and then obtain some practical fixed point theorems in the existing literature.

Theorem 7.1 Let $\varepsilon \in(0, \infty),(X, d)$ a complete $\varepsilon$-chainable metric space, $\left\{J_{q}\right\}_{q=1}^{\infty}$ be a sequence of multivalued mappings from $X$ into $C B(X)$ and $S: X \rightarrow X$ a surjection such that $0<d(S u, S v)<\varepsilon$, implies

$$
H\left(J_{q}(u), J_{r}(v)\right) \leq \mu(d(S u, S v)) d(S u, S v),
$$

$u, v \in X$, where $\mu:[0, \varepsilon) \rightarrow[0,1)$ is a MT-function, then $S$ and the sequence $\left\{J_{q}\right\}_{q=1}^{\infty}$ have a coincidence point, i.e., there exists $v^{*} \in X$ such that $S v^{*} \in \bigcap_{q \in \mathbb{N}}\left\{J_{q}\left(v^{*}\right)\right\}$.

Proof Define a sequence of $L$-fuzzy mappings $\left\{T_{q}\right\}_{q=1}^{\infty}$ from $X$ into $F_{L}(X)$ as, for some $\alpha_{L} \in$ $L \backslash\left\{0_{L}\right\}, T_{q}(v)(t)=\alpha_{L}$ if $t \in J_{q}(v)$ and $T_{q}(v)(t)=0_{L}$, otherwise. Then $\left[T_{q}(v)\right]_{\alpha_{L}}=J_{q}(v)$ for all $v \in X$, so $\left[T_{q}(v)\right]_{\alpha_{L}} \in C B(X)$ for all $v \in X$. Since

$$
D_{\alpha_{L}}\left(T_{q}(u), T_{r}(v)\right)=H\left(\left[T_{q}(u)\right]_{\alpha_{L}},\left[T_{r}(v)\right]_{\alpha_{L}}\right)=H\left(J_{q}(u), J_{r}(v)\right),
$$


for all $u, v \in X$, we deduce that condition (1) of Theorem 3.2 is satisfied for $\left\{T_{q}\right\}_{q=1}^{\infty}$. Hence there exists a point $v^{*}$ in $X$, such that $S v^{*} \in \bigcap_{q \in \mathbb{N}}\left[T_{q}\left(v^{*}\right)\right]_{\alpha_{L}}$. From this we conclude that $S v^{*} \in \bigcap_{q \in \mathbb{N}}\left\{J_{q}\left(v^{*}\right)\right\}$. This completes the proof.

Corollary 7.2 Let $\varepsilon \in(0, \infty),(X, d)$ a complete $\varepsilon$-chainable metric space, $\left\{J_{q}\right\}_{q=1}^{\infty}$ be a sequence of multivalued mappings from $X$ into $C B(X)$ and $S: X \rightarrow X$ a surjection such that $0<d(S u, S v)<\varepsilon$, implies

$$
H\left(J_{q}(u), J_{r}(v)\right) \leq \rho d(S u, S v)
$$

$u, v \in X$, where $\rho \in(0,1)$, then $S$ and the sequence $\left\{J_{q}\right\}_{q=1}^{\infty}$ have a coincidence point, i.e., there exists $v^{*} \in X$ such that $S v^{*} \in \bigcap_{q \in \mathbb{N}}\left\{J_{q}\left(v^{*}\right)\right\}$.

By taking $S=I$ in Theorem 7.1 we get the following.

Corollary 7.3 [25] Let $\varepsilon \in(0, \infty],(X, d)$ be a complete $\varepsilon$-chainable metric space, and $\left\{J_{q}\right\}_{q=1}^{\infty}$ be a sequence of multivalued mappings from $X$ into $C B(X)$ such that $0<d(u, v)<\varepsilon$, implies

$$
H\left(J_{q}(u), J_{r}(v)\right) \leq \mu(d(u, v)) d(u, v)
$$

$u, v \in X$, where $\mu:[0, \varepsilon) \rightarrow[0,1)$ is a MT-function, then the sequence $\left\{J_{q}\right\}_{q=1}^{\infty}$ has a common fixed point, i.e., there exists $v^{*} \in X$ such that $v^{*} \in \bigcap_{q \in \mathbb{N}}\left\{J_{q}\left(v^{*}\right)\right\}$.

Corollary 7.4 $[9,11,13]$ Let $(X, d)$ be a complete metric space, J a multivalued mapping from $X$ into $C B(X)$, and $\mu:[0, \infty) \rightarrow[0,1)$ a MT-function such that

$$
H(J u, J v) \leq \mu(d(u, v)) d(u, v)
$$

for all $u, v \in X$. Then $J$ has a fixed point in $X$.

Proof Taking $q=r=1$ with $\varepsilon=\infty$, in the above corollary we get the required result.

Corollary 7.5 [26] Let $\varepsilon \in(0, \infty],(X, d)$ a complete $\varepsilon$-chainable metric space and $J$ be a multivalued mapping from $X$ into $C B(X)$ such that $0<d(u, v)<\varepsilon$, implies

$$
H(J(u), J(v)) \leq \rho d(u, v)
$$

$u, v \in X$, where $\rho \in(0,1)$. Then J has a fixed point.

By considering $J$ to be a single valued mapping in the above corollary we deduce the following result.

Corollary 7.6 [17] Let $\varepsilon \in(0, \infty],(X, d)$ a complete $\varepsilon$-chainable metric space and $T: X \rightarrow$ $X$ be a $(\varepsilon, \lambda)$ uniformly locally contractive single valued mapping. Then $T$ has a fixed point. 


\section{Applications to integral and differential equations}

The theory of differential inclusions was scientifically recognized by Aubin and Cellina [28]. They studied the existence and properties of solutions to differential inclusions of the form $\frac{d}{d t} x(t) \in L(t, x(t))$. Theorem 3.2 can deal with the existence of the solutions of differential inclusions of form $\frac{d}{d t} f(x(t)) \in K(t, x(t))$. However, to identify it we have to explore some extra material concerned with a version of measurable selection theorem for continuous multivalued functions with nonempty convex closed (or compact) values on a Banach space, which may be problematic for a common reader. Therefore in this section, let us restrict our research area. We shall mainly consider the nonlinear differential equations of form $\frac{d}{d t} f(x(t))=K(t, x(t))$. The main objective of this section is to study the existence and uniqueness of the solution of a general class of Volterra integral equations arising from differential equations of the form $\frac{d}{d t} f(x(t))=K(t, x(t))$ under various assumptions on the functions involved. Theorem 3.2 together with a function space $(C[a, b], \mathbb{R})$, and a contractive inequality are used to establish the result. Consider the integral equation:

$$
h(x(t))-\eta \int_{a}^{t} L(s, x(s)) d s=g(t)
$$

where $x:[a, b] \rightarrow \mathbb{R}$ is unknown, and $h: \mathbb{R} \rightarrow \mathbb{R}$ is given, $\eta$ is a parameter. If $h=I$ (the identity mapping on $\mathbb{R}$ ), then (3) is known as the Volterra integral equation.

Theorem 8.1 Let $L_{0}:[a, b] \rightarrow \mathbb{R}, L:[a, b] \times \mathbb{R} \rightarrow \mathbb{R}$ be continuous mappings and $h: \mathbb{R} \rightarrow$ $\mathbb{R}$ a continuous surjection. If there exists $K<\frac{1}{b-a}$ such that for $r, s \in \mathbb{R}$,

$$
|h r-h s|<\varepsilon \quad \Longrightarrow \quad|L(t, r)-L(t, s)| \leq K|h r-h s|, \quad t \in[a, b],
$$

then the integral equation

$$
h(x(t))=L_{0}(t)+\int_{a}^{t}[L(s, x(s))] d s, \quad t \in[a, b]
$$

has a solution in $(C[a, b], \mathbb{R})$.

Proof Let $X=(C[a, b], \mathbb{R})$; then $X$ is a complete $\varepsilon$-chainable metric space for $\varepsilon \in(0, \infty)$. Let $\varphi_{L}: X \rightarrow L \backslash\left\{0_{L}\right\}$ be an arbitrary mapping. Define $d: X \times X \rightarrow \mathbb{R}$ as $d(x, y)=$ $\max _{t \in[a, b]}|x(t)-y(t)|$. Assume that, for $x \in X$,

$$
\tau_{x}(t)=L_{0}(t)+\int_{a}^{t}[L(s, x(s))] d s, \quad \text { for all } t \in[a, b] .
$$

Define the mappings $T: X \rightarrow F_{L}(X)$ and $S: X \rightarrow X$ as follows:

$$
\begin{aligned}
& T(x)(f)= \begin{cases}\varphi_{L}(x) & \text { if } f(t)=\tau_{x}(t) \text { for all } t \in[a, b], \\
0_{L} & \text { otherwise, }\end{cases} \\
& S(x)(t)=h(x(t)) \text { for all } t \in[a, b] .
\end{aligned}
$$

Note that $|h x(t)-h y(t)|<\varepsilon$ for all $t \in[a, b] \Longleftrightarrow d(S x, S y)<\varepsilon$. 
Take $\alpha_{L}=\varphi_{L}(x)$. Moreover, for some $f \in[T x]_{\alpha_{L}}$, we obtain, $T(x)(f)=\varphi_{L}(x)$. Then, by the assumptions, for every $f \in X$ there exists $y \in X$ such that $f=S y=h \circ y$.

Moreover, we obtain

$$
D_{\alpha_{L}}(T x, T y)=\max _{t \in[a, b]}\left|\tau_{x}(t)-\tau_{y}(t)\right| .
$$

If $|h x(t)-h y(t)|<\varepsilon$ for all $t \in[a, b]$, by assumptions, we have

$$
\begin{aligned}
\left|\tau_{x}(t)-\tau_{y}(t)\right| & =\left|\int_{a}^{t}[L(s, x(s))] d s-\int_{a}^{t}[L(s, y(s))] d s\right| \\
& \leq \int_{a}^{t}|L(s, x(s))-L(s, y(s))| d s \\
& \leq \int_{a}^{t} K|h(x(s))-h(y(s))| d s \\
& \leq K\left(\sup _{t \in[a, b]}|(h x)(t)-(h y)(t)|\right)\left|\int_{a}^{t} d s\right| \\
& \leq K(b-a) d(h \circ x, h \circ y) \\
& \leq K(b-a) d(S x, S y) .
\end{aligned}
$$

It implies that

$$
D_{\alpha_{L}}(T x, T y) \leq K(b-a) d(S x, S y) .
$$

Hence, if for a $M T$-function $\mu:[0, \varepsilon) \rightarrow[0,1), \mu(d(S x, S y))=K(b-a)$, all conditions of Theorem 3.2 are satisfied to find a continuous function $u:[a, b] \rightarrow \mathbb{R}$ such that $\mathrm{Su} \in$ $[T(u)]_{\alpha_{L}}$. That is, $h \circ u=\tau_{u}$ and $u$ will be a solution of the integral equation (4).

Corollary 8.2 Let $K_{0} \in \mathbb{R}, L:[a, b] \times \mathbb{R} \rightarrow \mathbb{R}$ are a continuous mapping and $h: \mathbb{R} \rightarrow \mathbb{R} a$ continuous surjection. If there exists $K<\frac{1}{b-a}$ such that for $r, s \in \mathbb{R}$,

$$
|h r-h s|<\varepsilon \quad \Longrightarrow \quad|L(t, r)-L(t, s)| \leq K|h r-h s|, \quad t \in\left[t_{0}, t_{0}+\delta\right]
$$

then the initial value problem

$$
\frac{d}{d t} h(x(t))=L(s, x(t)), \quad h\left(x\left(t_{0}\right)\right)=K_{0}, \quad t \in\left[t_{0}, t_{0}+\delta\right]
$$

has a solution in $(C[a, b], \mathbb{R})$.

Proof Considering the integral equation:

$$
h(x(t))=K_{0}+\int_{t_{0}}^{t}[L(s, x(s))] d s, \quad t \in\left[t_{0}, t_{0}+\delta\right],
$$

we get the required result by Theorem 8.1 for $L_{0}(t)=K_{0}$. 


\section{An illustrative example}

In this section we provide a simple but practical example to illustrate the theory developed in the above section. The problem under consideration is a solution of the nonlinear integral equation:

$$
x^{7}(t)-\tan t=\int_{0}^{t}\left[x^{7}(s)+17 s+5\right] s d s, \quad t \in[0, c], c<1 .
$$

Note that, for $L(t, s)=\left[x^{7}(s)+17 s+5\right] s, L_{0}(t)=\tan t, h s=s^{7}$,

$$
|L(t, r)-L(t, s)| \leq c|h r-h s|
$$

for all $t \in[0, c]$ and all conditions of Theorem 8.1 are satisfied (for $K=c, a=0$ ). Let $X=$ $(C[0, c], \mathbb{R})$. Define the mappings $T: X \rightarrow F_{L}(X)$ and $S: X \rightarrow X$ as follows:

$$
\begin{aligned}
& T(x)(f)= \begin{cases}\varphi_{L}(x) & \text { if } f(t)=\tau_{x}(t) \text { for all } t \in[0, c], \\
0_{L} & \text { otherwise, }\end{cases} \\
& S x=x^{7} .
\end{aligned}
$$

In the following we approximate the value of $u$, by constructing the iterative sequences:

$$
x_{n+1} \in(C[0, c], \mathbb{R}), \quad S x_{n+1} \in\left[T x_{n}\right]_{\alpha_{L}} \quad \text { for some } \alpha_{L} \in L \backslash\left\{0_{L}\right\} .
$$

Suppose

$$
\tau_{x}(t)=\tan t+\int_{0}^{t}\left[x^{7}(s)+17 s+5\right] s d s, \quad \text { for all } t \in[0, c] .
$$

Note that

$$
[T x]_{\varphi_{L}(x)}=\left\{f \in X: T(x)(f)=\varphi_{L}(x)\right\}=\left\{\tau_{x}\right\} .
$$

Let $x_{0}:[0, c] \rightarrow \mathbb{R}$ be defined as $x_{0}(t)=0$ for all $t \in[0, c]$. Then

$$
\left[T x_{0}\right]_{\varphi_{L}(x)}=\left\{\tau_{x_{0}}\right\} .
$$

Thus, $S x_{1}=\tau_{x_{0}}$, where

$$
\begin{aligned}
& \tau_{x_{0}}(t)=\tan t+\int_{0}^{t}[17 s+5] s d s=\tan t+\frac{17 t^{3}}{3}+\frac{5 t^{2}}{2} \text { and } \\
& x_{1}(t)=\sqrt[7]{\tan t+\frac{17 t^{3}}{3}+\frac{5 t^{2}}{2}} .
\end{aligned}
$$

Now,

$$
S x_{2}=\tau_{x_{1}} \in\left[T x_{1}\right]_{\varphi_{L}(x)},
$$


where

$$
\begin{aligned}
\tau_{x_{1}}(t) & =\tan t+\int_{0}^{t}\left(\left(\tan t+\frac{17 s^{3}}{3}+\frac{5 s^{2}}{2}\right)+17 s+5\right) s d s \\
& =\tan t+\frac{17 t^{3}}{3}+\frac{5 t^{2}}{2}+\left(\frac{17 t^{5}}{3.5}+\frac{5 t^{4}}{2.4}+\frac{(\tan t) t^{2}}{2}\right)
\end{aligned}
$$

and

$$
x_{2}(t)=\sqrt[7]{\tan t+\frac{17 t^{3}}{3}+\frac{5 t^{2}}{2}+\left(\frac{17 t^{5}}{3.5}+\frac{5 t^{4}}{2.4}+\frac{(\tan t) t^{2}}{2}\right)} .
$$

Similarly,

$$
\begin{aligned}
S x_{3}= & \tau_{x_{2}}(t)=\tan t \\
& +\int_{0}^{t}\left(\left(\tan t+\frac{17 t^{3}}{3}+\frac{5 t^{2}}{2}+\frac{1}{27}\left(\frac{17 t^{5}}{3.5}+\frac{5 t^{4}}{2.4}+\frac{(\tan t) t^{2}}{2}\right)\right)+17 s+5\right) s d s \\
= & \tan t+\frac{17 t^{3}}{3}+\frac{5 t^{2}}{2}+\left(\frac{17 t^{5}}{3.5}+\frac{5 t^{4}}{2.4}+\frac{(\tan t) t^{2}}{2}\right) \\
& +\left(\frac{17 t^{7}}{3.5 .7}+\frac{5 t^{6}}{2.4 .6}+\frac{(\tan t) t^{4}}{2.4}\right)
\end{aligned}
$$

and $x_{3}(t)=\left(\tan t+\frac{17 t^{3}}{3}+\frac{5 t^{2}}{2}+\left(\frac{17 t^{5}}{3.5}+\frac{5 t^{4}}{2.4}+\frac{(\tan t) t^{2}}{2}\right)+\left(\frac{17 t^{7}}{3.5 .7}+\frac{5 t^{6}}{2.4 .6}+\frac{(\tan t) t^{4}}{2.4}\right)\right)^{\frac{1}{7}}$.

It follows that

$$
\begin{aligned}
& \lim _{n \rightarrow \infty} S x_{n}= \tan t+\tan t \sum_{n=1}^{\infty}\left(\frac{t^{2 n}}{(2 n)(2 n-2) \cdots 2}\right) \\
&+\sum_{n=1}^{\infty}\left(\frac{17 t^{2 n+1}}{(2 n+1)(2 n-1) \cdots 3}+\frac{5 t^{2 n}}{(2 n)(2 n-2) \cdots 2}\right) \\
& \in[T u]_{\varphi_{L}(x)} .
\end{aligned}
$$

Hence,

$$
\begin{aligned}
u(t)= & \left(\tan t+\tan t \sum_{n=1}^{\infty}\left(\frac{t^{2 n}}{(2 n)(2 n-2) \cdots 2}\right)\right. \\
& \left.+\sum_{n=1}^{\infty}\left(\frac{17 t^{2 n+1}}{(2 n+1)(2 n-1) \cdots 3}+\frac{5 t^{2 n}}{(2 n)(2 n-2) \cdots 2}\right)\right)^{\frac{1}{7}} \\
= & \tan t+(5+\tan t) t^{2} e^{\frac{t^{2}}{2}}-t+\sqrt{\frac{\pi}{2}} e^{\frac{t^{2}}{2}} \operatorname{erf}\left(\frac{t}{\sqrt{2}}\right)
\end{aligned}
$$

is a solution of integral equation (6). 
Authors' contributions

All authors contributed equally and significantly in writing this article. All authors read and approved the final manuscript.

\section{Author details}

'Department of Mathematics, International Islamic University, H-10, Islamabad, 44000, Pakistan. ${ }^{2}$ Department of Mathematics, King Abdul Aziz University, Jeddah, Saudi Arabia. ${ }^{3}$ Department of Mathematics, COMSATS Institute of Information Technology, Chack Shahzad, Islamabad, 44000, Pakistan.

\section{Acknowledgements}

Authors are grateful to the referees for their valuable suggestions and critical remarks for improving this paper.

Received: 20 June 2014 Accepted: 24 September 2014 Published: 14 Oct 2014

\section{References}

1. Zadeh, LA: Fuzzy sets. Inf. Control 8, 338-353 (1965)

2. Heilpern, S: Fuzzy mappings and fixed point theorems. J. Math. Anal. Appl. 83, 566-569 (1981)

3. Azam, A, Beg, I: Common fixed points of fuzzy maps. Math. Comput. Model. 49, 1331-1336 (2009)

4. Azam, A, Arshad, M, Beg, I: Fixed points of fuzzy contractive and fuzzy locally contractive maps. Chaos Solitons Fractals 42, 2836-2841 (2009)

5. Azam, A, Arshad, M, Vetro, P: On a pair of fuzzy $\phi$-contractive mappings. Math. Comput. Model. 52, $207-214$ (2010)

6. Azam, A, Arshad, M: A note on 'Fixed point theorems for fuzzy mappings' by P Vijayaraju and M Marudai. Fuzzy Sets Syst. 161, 1145-1149(2010)

7. Azam, A, Waseem, M, Rashid, M: Fixed point theorems for fuzzy contractive mappings in quasi-pseudo-metric spaces. Fixed Point Theory Appl. 2013, Article ID 27 (2013). doi:10.1186/1687-1812-2013-27

8. Du, WS: On coincidence point and fixed point theorems for nonlinear multivalued maps. Topol. Appl. 159, 49-56 (2012)

9. Hu, T: Fixed point theorems for multivalued mappings. Can. Math. Bull. 23, 193-197 (1980)

10. Kamran, T: Common fixed points theorems for fuzzy mappings. Chaos Solitons Fractals 38, 1378-1382 (2008)

11. Mizoguchi, N, Takahashi, W: Fixed point theorems for multi-valued mappings on complete metric spaces. J. Math. Anal. Appl. 188, 141-177 (1989)

12. Paul, S, Tripathy, BC, Das, NR: A fixed point theorem in a generalized fuzzy metric space. Bol. Soc. Parana. Mat. 32(2), 221-227 (2013)

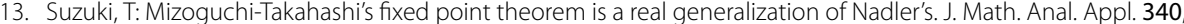
752-755 (2008)

14. Tripathy, BC, Baruah, A, Et, M, Gungor, M: On almost statistical convergence of new type of generalized difference sequence of fuzzy numbers. Iran. J. Sci. Technol., Trans. A, Sci. 36(2), 147-155 (2012)

15. Tripathy, BC, Ray, GC: On mixed fuzzy topological spaces and countability. Soft Comput. 16, 1691-1695 (2012)

16. Tripathy, BC, Paul, S, Das, NR: Banach's and Kannan's fixed point results in fuzzy 2-metric spaces. Proyecciones 32(4), 363-379 (2013)

17. Edelstein, M: An extension of Banach's contraction principle. Proc. Am. Math. Soc. 12, 7-12 (1961)

18. Ahmad, J, Azam, A, Romaguera, S: On locally contractive fuzzy set-valued mappings. J. Inequal. Appl. 2014, Article ID 74 (2014)

19. Arshad, M, Azam, A: Fixed points solutions of sequence of locally contractive fuzzy mappings via iterative process. In: International Conference of Mathematical Sciences (ICM 2012), 11-14 March, Al Ain, UAE (2012)

20. Hu, T, Rosen, H: Locally contractive and expansive mappings. Proc. Am. Math. Soc. 86, 656-662 (1982)

21. Ko, HM, Tasi, YH: Fixed point theorems for localized property. Tamkang J. Math. 8, 81-85 (1977)

22. Kuhfitting, PK: Fixed point of locally contractive and nonexpansive set valued mappings. Pac. J. Math. 65, 399-403 (1976)

23. Goguen, JA: L-Fuzzy sets. J. Math. Anal. Appl. 18, 145-174 (1967)

24. Rashid, M, Azam, A, Mehmood, N: L-Fuzzy fixed points theorems for L-fuzzy mappings via $\beta_{\mathcal{F}_{L}}$-admissible pair. Sci. World J. (2014). doi:10.1155/2014/853032

25. Beg, I, Azam, A: Fixed points of multivalued locally contractive mappings. Boll. Unione Mat. Ital., A (7) 4, 227-233 (1990)

26. Nadler, SB: Multivalued contraction mappings. Pac. J. Math. 30, 475-488 (1969)

27. Du, WS: Coupled fixed point theorems for nonlinear contractions satisfied Mizoguchi-Takahashi's condition in quasi-ordered metric spaces. Fixed Point Theory Appl. 2010, Article ID 876372 (2010). doi:10.1155/2010/876372

28. Aubin, JP, Cellina, A: Differential Inclusions. Springer, Berlin (1984)

10.1186/1687-1812-2014-212

Cite this article as: Rashid et al.: Coincidence theorems via alpha cuts of $L$-fuzzy sets with applications. Fixed Point Theory and Applications 2014, 2014:212 